\title{
Avaliação das condições ambientais no laboratório de anatomia patológica de um hospital universitário no município do Rio de Janeiro
}

\author{
Assessment of environmental conditions in the pathological anatomy laboratory of a university hospital located \\ in Rio de Janeiro
}

Sheila de Lira Franklin'; Déa Regina Bettini²; Ubirajara Aluizio de Oliveira Mattos³ Júlio Domingos Nunes Fortes ${ }^{4}$

\section{unitermos \\ Saúde do trabalhador \\ Higiene do trabalho \\ Riscos ambientais \\ Mapa de riscos \\ Laboratórios de anatomia patológica}

\section{resumo}

Introdução: Em laboratórios de anatomia patológica existem diversos tipos de riscos ambientais e ocupacionais. Tais riscos podem estar associados a acidentes do trabalho ou doenças ocupacionais. Objetivos: Identificar e avaliar os riscos do processo de trabalho, estimulando a percepção dos mesmos pelos trabalhadores. Métodos: Foi realizado o levantamento dos processos, fluxos de serviços, equipamentos, instalações, produtos, materiais, resíduos, equipes de trabalho e atividades dos trabalhadores. A seguir foram realizadas as medições dos agentes, elaborando-se a representação gráfica do mapa de riscos. Resultados: Observou-se a presença de riscos químicos, físicos, biológicos, acidentários e inadequações ergonômicas. Conclusão: Os riscos de natureza química e biológica foram identificados como principais no processo de trabalho. Também foi verificada a necessidade de reformas e maiores investimentos na aquisição de equipamentos de proteção individual e coletiva visando à melhoria das condições de trabalho. A adesão a normas de biossegurança e a inserção de programas de educação continuada são indispensáveis no processo de redução dos riscos ambientais e ocupacionais.

\section{abstract}

Background: In pathological anatomy laboratories, there are several kinds of environmental and occupational hazards, which can be associated with work accidents and occupational diseases. Objectives: To identify and assess work process risks in order to raise workers' awareness. Methods: It was carried out an investigation into work processes, flow of services, equipment, site, products, materials, residues, work teams and workers' activities. Subsequently, the agents were assessed and the graphic representation of the risk map was developed. Results: The presence of chemical, physical, biological and accidental risks and ergonomic inadequacies were observed. Conclusion: Biological and chemical agents were identified as the main risks in the work process. Furthermore, the necessity of refurbishment and additional investments in the purchase of individual and collective protection equipment was noticed. The adherence to biosecurity protocols and the introduction of continuing education programs are invaluable to reduce occupational and environmental hazards.

\section{key words}

Workers health

Occupational hygiene

Environmental risks

Risk map

Pathological anatomy laboratory

1. Mestra em Engenharia Ambiental; doutoranda em Meio Ambiente pela Universidade do Estado do Rio de Janeiro (UER)).

2. Mestra em Engenharia Ambiental pela UERJ.

3. Doutor em Arquitetura e Urbanismo pela Universidade de São Paulo (USP); professor titular da Faculdade de Engenharia da UER).

4. Doutor em Saúde Pública pela Escola Nacional de Saúde Pública (ENSP); professor adjunto da Faculdade de Engenharia da UER).

Trabalho realizado pelo Departamento de Engenharia Sanitária e de Meio Ambiente - Programa de Pós-Graduação em Engenharia Ambiental (PEAMB) da UER). 


\section{Introdução}

As atividades realizadas em laboratórios de anatomia patológica (LAP) são indispensáveis para o correto diagnóstico de doenças e o direcionamento de tratamento a ser ministrado a cada paciente ${ }^{(16)}$. Assim como em qualquer atividade laboral, os trabalhadores de LAP são expostos aos riscos inerentes ao seu processo de trabaIho, que podem ser: biológicos, químicos, físicos, acidentários e inadequações do ponto de vista ergonômico ${ }^{(17)}$.

O mapa de risco constitui metodologia importante para o reconhecimento dos riscos existentes em um local de trabalho(15). O ideal é que a elaboração desse mapeamento seja realizada pelos trabalhadores junto a especialistas da área, possibilitando dessa forma maiores participação e sensibilização dos trabalhadores sobre os problemas e as necessidades de intervenção. Entretanto, nem sempre é possível realizar mapas de risco com a participação dos trabalhadores.

A realização de avaliações ambientais está intimamente relacionada com a adoção de princípios de biossegurança, a qual abrange recomendações para riscos físicos, químicos, biológicos, ergonômicos e acidentários ${ }^{(12)}$.

De acordo com o item 9.1.5 da Norma Regulamentadora (NR) no 9, da Portaria no 3.214/78, consideram-se riscos ambientais os agentes físicos, químicos e biológicos existentes nos ambientes de trabalho que, em função de sua natureza, concentração ou intensidade e tempo de exposição, são capazes de causar danos à saúde do trabalhador $^{(4)}$.

A literatura nacional e estrangeira tem apresentado estudos de condições de trabalho em laboratórios de análises e pesquisas clínicas, patologia clínica e congêneres. Entretanto, constata-se carência de estudos relacionados com as condições de trabalho em LAP. Além disso, observa-se também a precariedade de legislações e normas pertinentes ao desenvolvimento da atividade de anatomia patológica, no que tange à biossegurança e à qualidade do ar interior ${ }^{(2)}$.

Entre os riscos existentes em LAP, destacam-se os químicos e biológicos. Este artigo apresenta resultados de pesquisa em LAP de hospital universitário localizado no município do Rio de Janeiro, onde foi realizada avaliação dos riscos ambientais e seus impactos na saúde dos trabalhadores. O LAP estudado apresenta sistema de climatização artificial e realiza atividades de ensino, pesquisa e extensão, bem como prestação de assistência ao hospital, por meio do diagnóstico das patologias dos seus pacientes.

\section{Metodologia}

Foram investigados riscos químicos, biológicos, físicos, ergonômicos e acidentários e construídos mapas de riscos. Para avaliação de agentes químicos (gases e vapores orgânicos), foram realizadas coletas e análises variáveis devido às especificidades dos contaminantes. A avaliação da qualidade do ar em laboratórios visava confirmar a hipótese sobre contaminação dos ambientes estudados, tendo em vista que a proposta de intervenção para melhoria da qualidade do ar justifica-se, principalmente, quando há esse tipo de contaminação.

A avaliação do ar para investigação dos riscos químicos e biológicos se deu em duas etapas, amostragem do ar e análise laboratorial das amostras.

A escolha do método de amostragem (com a devida aparelhagem) proporcionou a determinação da concentração dos contaminantes selecionados no ambiente.

A Agência Nacional de Vigilância Sanitária (ANVISA), subordinada ao Ministério da Saúde (MS), não apresenta padrão de amostragem para vapores orgânicos. Buscouse, portanto, nas normas internacionais, métodos de avaliação aplicáveis ao tipo de laboratório em questão.

Para amostragem e análise do xileno, foi utilizada metodologia com base nos procedimentos do Working Environment Measurement System in Japan (1991), Hygienist Technical Course (1985) e Segurança e Medicina do Trabalho (2001). A amostragem foi coletada com tubo de carvão ativado, bomba gravimétrica (SKC e Gil Air) e nebulizador. O método analítico utilizado foi a cromatografia gasosa, detecção por ionização de chama (CG-DIC).

Para amostragem e análise do formaldeído, foi utilizada metodologia com base na United States Environmental Protection Agency (USEPA-TO-11A). As amostras foram coletadas com o cartucho C-18 impregnado com 2,4 dinitrofenilhidrazina (DNPH), bomba gravimétrica (SKC e Gil Air) e nebulizador. O método analítico utilizado foi a cromatografia líquida de alta eficiência (CLAE).

Quanto às amostras de micro-organismos, a avaliação quantitativa foi realizada com base nos parâmetros estabelecidos pela Resolução (RE) no 9, de 01/2003, da 
ANVISA para ambientes climatizados artificialmente. Essa legislação, entretanto, é a única existente sobre qualidade de ar em ambientes climatizados e os valores foram utilizados como base comparativa.

Para realização das amostragens dos agentes biológicos foi utilizado o coletor Andersen de 1 estágio e placas de Petri devidamente preparadas com meios de cultura específicos para o crescimento e o desenvolvimento de fungos e bactérias. O material, depois desse processo, foi encaminhado para incubação em estufa. As bactérias foram incubadas à temperatura de $350^{\circ} \mathrm{C}$ enquanto os fungos, a $250^{\circ} \mathrm{C}$, ambos por um período de sete dias. Transcorridos esses dias de cultura, foi realizada a contagem do número de colônias formadas por $\mathrm{m}^{3}\left(\mathrm{ufc} / \mathrm{m}^{3}\right)$.

Para realização das amostragens dos agentes físicos foram utilizados três diferentes equipamentos (Termômetro Questemp0 10, Area Heat Stress Monitor - Quest Technologies; Decibelímetro ONO SOKKI LA - 220S, Integrating Sound Level Meter e Luxímetro Lux Meter ANA-315, Tokyo Photo-Eletric CO LTD).

Além de todos os equipamentos relacionados, foram realizadas visitas ao local, a fim de identificar riscos acidentários e propor medidas corretivas que possibilitassem melhorias na qualidade ambiental. Durante a realização da pesquisa, também foram levantados os acidentes já ocorridos. No entanto, foi constatado que a comunicação de acidentes de trabalho era rara.

\section{Resultados}

Com relação aos riscos físicos, a temperatura foi medida de acordo com as variáveis: índice de bulbo úmido e termômetro de globo (IBUTG), temperaturas de globo, bulbo seco e bulbo úmido, a fim de constatar se os valores correspondiam às exigências das legislações vigentes, de acordo com o Anexo no 3, da NR-15 e com as indicações previstas nas Tabelas $\mathbf{1}$ e $\mathbf{2}$, sobre limites de tolerância para exposição ao calor, considerando o IBUTG máximo indicado. Os processos de trabalho realizados no ambiente selecionado foram classificados como contínuos e leves, sendo o valor máximo indicado de $30^{\circ} \mathrm{C}$. Não foram registrados valores superiores aos limites definidos.

Tabela 1

Medições de agentes químicos

\begin{tabular}{|c|c|c|c|c|}
\hline \multirow{3}{*}{ Agente químico } & \multirow{3}{*}{ Locais } & \multirow{3}{*}{ Resultado } & \multicolumn{2}{|c|}{ *Situação } \\
\hline & & & Máximo & Mínimo \\
\hline & & & ppm & ppm \\
\hline \multirow{7}{*}{ Xileno } & Entrada do edifício & 0,07 & - & Conforme \\
\hline & Corredor & 0,26 & - & Conforme \\
\hline & $\begin{array}{l}\text { Sala de técnicas histológicas e } \\
\text { citológicas }\end{array}$ & 0,42 & - & Conforme \\
\hline & Sala de imunohistoquímica & 0,14 & - & Conforme \\
\hline & Sala de colorações especiais & 0,19 & - & Conforme \\
\hline & Auditório & 0,04 & - & Conforme \\
\hline & Corredor & 0,34 & - & Conforme \\
\hline \multirow{5}{*}{ Formaldeído } & $\begin{array}{l}\text { Sala de técnicas histológicas e } \\
\text { citológicas }\end{array}$ & 1,45 & 0,29 & Conforme \\
\hline & Sala de necropsia A & 1,56 & 0,92 & Conforme \\
\hline & Sala de necropsia B & $2,32^{* *}$ & 0,34 & Não conforme \\
\hline & Sala de clivagem & $6,21^{* *}$ & 2,25 & Não conforme \\
\hline & Auditório & 0,12 & 0,1 & Conforme \\
\hline
\end{tabular}

*Parâmetros estabelecidos pela n-15, Anexo nº 11, Portaria TEM n 3.214/78 Limite máximo de tolerância de 78 ppm para xileno e de 1,6 ppm para formaldeído. 


\section{Tabela 2 Medições de agentes biológicos}

\begin{tabular}{|c|c|c|c|c|}
\hline \multirow[b]{2}{*}{$\begin{array}{l}\text { Agentes } \\
\text { biológicos }\end{array}$} & \multirow[b]{2}{*}{ Locais } & \multirow[b]{2}{*}{ Resultados } & \multicolumn{2}{|c|}{ *Situação } \\
\hline & & & $\begin{array}{l}\text { Máximo } \\
\text { ufc/m3 }\end{array}$ & $\begin{array}{l}\text { Mínimo } \\
\text { ufc/m3 }\end{array}$ \\
\hline \multirow{5}{*}{ Bactérias } & $\begin{array}{l}\text { Sala de técnicas histológicas e } \\
\text { citológicas }\end{array}$ & 18 & 15 & Conforme \\
\hline & Sala de clivagem & 15 & - & Conforme \\
\hline & Auditório & 22 & - & Conforme \\
\hline & Corredor & 15 & - & Conforme \\
\hline & Arquivo morto & 13 & - & Conforme \\
\hline \multirow{5}{*}{ Fungos } & $\begin{array}{l}\text { Sala de técnicas histológicas e } \\
\text { citológicas }\end{array}$ & 28,3 & 14 & Conforme \\
\hline & Sala de clivagem & 70,7 & - & Conforme \\
\hline & Auditório & 49,9 & - & Conforme \\
\hline & Corredor & 10,6 & - & Conforme \\
\hline & Arquivo morto & 7,1 & - & Conforme \\
\hline
\end{tabular}

*Parâmetros estabelecidos pela Resolução n 9, de janeiro de 2003, da ANVISA, do MS, para ambientes climatizados artificialmente - Limite máximo permitido: $750 \mathrm{ufc} / \mathrm{m} 3$

ANVISA: Agência Nacional de Vigilância Sanitária; MS: Ministério da Saúde.

De acordo com a norma NBR no 5.413/1992, o ambiente deve apresentar nível de iluminância entre 300 e 750 Lux. Em todos os ambientes analisados (sala de necropsia, sala de clivagem, técnicas histológicas e citológicas) foram detectadas não conformidades com relação à iluminação, o que provoca maior esforço visual e fadiga nos trabalhadores, aumentando o risco de acidentes. Os valores máximos e mínimos de iluminância registrados nesses ambientes foram: sala de necropsia (415 e 110 Lux), sala de clivagem (230 e 100 Lux), sala de técnicas histológicas e citológicas (550 e 110 Lux).

O nível de pressão sonora foi medido e, de acordo com o Anexo oㅜ 1, limites de tolerância para ruído contínuo ou intermitente, da NR-15, Portaria no 3.214/78, os valores registrados encontravam-se abaixo desse limite, fixado para o ambiente, que é de $85 \mathrm{~dB}(\mathrm{~A})$ para oito horas diárias. $O$ valor máximo foi registrado na sala de necropsia $(75,7 \mathrm{~dB}(A))$ e o mínimo, na sala de clivagem $(66,1 \mathrm{~dB}(\mathrm{~A}))$.

Os principais riscos de acidentes identificados corresponderam à manipulação de materiais perfurocortantes, arranjo físico inadequado e risco de incêndio e explosões devido à estocagem de material químico na sala.

Com relação aos riscos ergonômicos, foram detectados como principais, postura inadequada nas atividades em mesas e bancadas, longa permanência na postura sentada, monotonia e repetitividade em atividades em geral. Tais fatores contribuem para incidência de dores musculares e de coluna, tendinite, bursite, irritação e estresse.

Entre os agentes químicos encontrados em LAP, os que apresentaram maior risco à saúde, tanto pela gravidade quanto pela frequência de uso e concentração, foram o formaldeído e o xileno. Foram realizadas medições de formaldeído e xileno em pontos variados. As concentrações detectadas para xileno e formaldeído podem ser observadas na Tabela 1.

Com relação à avaliação de riscos biológicos veiculados pelo ar, nenhum valor detectado foi superior aos limites definidos pela legislação vigente; os resultados desta medição são apresentados na Tabela 2.

\section{Discussão}

As atividades desenvolvidas em um LAP requerem extrema atenção e concentração. As posturas de trabaIho são variáveis. Na sala de clivagem os trabalhadores permanecem sentados por muito tempo e na sala de necropsia permanecem muito tempo em pé. De uma forma geral as atividades são repetitivas.

Embora não existissem registros oficiais por meio de protocolos de ocorrência, houve relatos de trabalha- 
dores que já se acidentaram. No ambiente há um risco frequente de ferimento por material perfurocortante e contaminação por agentes patogênicos, além do risco de queda de frascos, vidros e outros materiais. As prateleiras se encontravam em mau estado de conservação com risco de tombamento pelo excesso de peso. A situação sugere necessidade de manutenção contínua das prateleiras.

A investigação concluiu que os maiores riscos ambientais eram de natureza química e biológica. Com relação aos riscos químicos, as concentrações de xileno verificadas foram comparadas com o limite de tolerância estabelecido pela NR 15, Anexo no 11 da Portaria no $3.214 / 78$, encontrando-se abaixo desse valor.

$\mathrm{Na}$ sala de técnicas citológica e histológica, foram encontradas as maiores concentrações de xileno com relação às demais, devido ao uso das cubas das baterias de colorações e autotécnicos que ficam destampados, possibilitando a evaporação dos compostos voláteis (xilol, álcool e formol) para o ar.

Os níveis detectados de xileno nas salas estudadas foram considerados baixos quando comparados com os limites de tolerância (LT) adotados pela NR 15, Anexo no 11 (78 ppm), pela Conferência Americana de Higienistas Industriais Governamentais (ACGIH) (100 ppm), pela Occupational Safety \& Health Administration (OSHA) (100 ppm) e pela National Institute for Occupational Safety and Health (NIOSH) (100 ppm). Esse fato não exclui a necessidade de busca de intervenções para melhoria do ar com vistas à proteção da saúde dos trabalhadores. Segundo a NR 7, mesmo que os resultados tenham apontado baixas concentrações, a simples constatação da presença dos contaminantes implica a tomada de medidas preventivas e o monitoramento biológico.

Quanto às concentrações de formaldeído, foi verificado que os valores encontrados em todos os pontos ultrapassaram o limite de exposição da NIOSH de 0,016 ppm. Além disso, nove dos 15 valores medidos ultrapassaram o limite de exposição da OSHA de 0,75 ppm, ambos para $40 \mathrm{~h}$ semanais de exposição. Cinco concentrações ultrapassaram o LT da NR 15, de 1,6 ppm. Lembramos que, para a NIOSH, o valor Ceiling (valor teto), que não pode ser ultrapassado em nenhum momento da jornada de trabalho, é de 0,1 ppm.

Alguns dos valores revelaram uma situação alarmante: as concentrações correspondentes a 4,58 ppm e 6,21 ppm ultrapassam o valor máximo estabelecido pela NR 15, caracterizando situação de risco grave e iminente.
Isso implicaria a interdição da sala e a paralisação das atividades nela exercidas até que medidas de controle dos níveis de formaldeído fossem implementadas.

Na sala de necropsia, todas as concentrações de formaldeído encontradas estavam abaixo do LT da NR 15, as quais variaram de 1,55 ppm a 0,91 ppm. Os valores encontrados são considerados concentrações elevadas quando comparadas aos padrões de exposição da NIOSH e da OSHA. Além disso, as medições se realizaram sem que houvesse procedimentos de necropsia.

As concentrações de 6,21 ppm e 4,58 ppm encontradas na sala de clivagem foram as mais elevadas em todo o LAP, devido aos diversos frascos de tecidos e à solução de formaldeído, que vem a ser a fonte principal e difusa de contaminação química. Devido à frequência de uso dessa sala, o risco químico é o mais elevado em todo o setor.

Não foram encontrados, na legislação brasileira, parâmetros de concentrações de xileno e formaldeído específicos para laboratórios de exame de elementos anormais e sedimentoscopia (EAS) e LAP, entre outros. Atualmente, adotam-se os padrões estabelecidos pelo Ministério do Trabalho e Emprego (MTE), por meio da Portaria no $3.214 / 78$, NR 15, Anexo oo 11, a saber: 100 ppm para xileno e 1,6 ppm para formaldeído. Esses padrões, contudo, podem não refletir a realidade de laboratórios que, devido a diferentes processos, possuem peculiaridades.

O MS, pela Portaria no 3.523/1998 e da RE no 9/2003, especifica que nos ambientes climatizados de uso restrito, com exigências de filtros absolutos ou instalações especiais, tais como os que atendem a instalações hospitalares, sejam aplicadas as normas e os regulamentos específicos. A RE no 9/2003 não traz uma norma técnica para avaliação de compostos orgânicos, e os valores máximos de referência de parâmetros de qualidade do ar, que não os limites de tolerância, como umidade relativa, número de trocas de ar por hora, por pessoa, não são aplicáveis aos ambientes hospitalares. Isso porque essa norma determina que esses ambientes devem seguir normas e regulamentos específicos. Vale ressaltar que tais normas e regulamentos não foram encontrados na legislação.

Para as substâncias químicas, xileno e formaldeído, a principal via de exposição é a respiratória. Mais de $90 \%$ do formaldeído inalado é absorvido no trato respiratório superior, segundo a Agência Internacional de Pesquisa sobre o Câncer (IARC) (2005), o que não descarta a penetração pela via dérmica, no caso do formaldeído, ou oral em ambos os casos. 
Segundo o Registro de Efeitos Tóxicos de Substâncias Químicas (RTECS), referido pela NIOSH, três classificações toxicológicas são aplicáveis ao xileno: é considerado irritante primário, afeta a reprodução e pode causar tumores benignos. A IARC classificou o xileno como pertencente ao Grupo 3, apresentando evidência humana e animal inadequada para classificá-lo cancerígeno, na revisão de 1999. Já a revisão de 2005 afirma que o xileno "não pode ser classificado como carcinogênico humano".

Com relação ao formaldeído, o RTECS assim o classifica: tumorigênico, mutagênico, irritante primário e afeta a reprodução, entre outras características.

Segundo documento da Agência de Proteção Ambiental Americana (EPA), o formaldeído atua como irritante do tecido conjuntivo do trato respiratório superior. Os sintomas são temporários e dependem do nível e da duração da exposição, podendo ocasionar ardência nos olhos, nariz, garganta, aperto no peito e respiração difícil. Reações agudas severas ao formaldeído (que tem um odor peculiar pungente) podem estar associadas à hiperssensibilidade.

Altas concentrações de formaldeído podem causar reações asmáticas pelo mecanismo de irritação. O formaldeído é uma das causas mais comuns de dermatite de contato e é considerado um sensibilizador da pele.

Quanto aos efeitos genéticos, há evidências de que o formaldeído é genotóxico, tanto em modelos in vitro quanto em cobaias e pessoas expostas. Em uma importante revisão executada pela IARC, em setembro de 2004, o formaldeído foi transferido para o Grupo 1, que corresponde a substâncias que foram comprovadas como sendo carcinógenas para humanos.

Com relação aos riscos biológicos, a literatura aponta que os maiores riscos de exposição e contaminação veiculada pelo ar em LAP dizem respeito aos procedimentos de necropsia ${ }^{(10)}$. Isso explicaria a mínima detecção para agentes biológicos, já que não foi possível realizar a medição com exposição de trabalhadores em procedimento. Vale ressaltar que alguns fungos e bactérias são altamente patogênicos e, independente da quantidade, podem afetar a saúde do ser humano. A suscetibilidade à contaminação está intimamente relacionada com o estado de imunocomprometimento, sendo, portanto, questão importante a ser observada à saúde dos trabaIhadores e às potenciais fontes de agentes biológicos. Nesse ambiente as principais vias de contaminação por agentes biológicos são a dérmica e a respiratória.
Com relação aos riscos biológicos, os valores encontraram-se abaixo do recomendado. Esse resultado não indicou necessariamente que no ambiente não existiam riscos de contaminação biológica no ar, pois as análises qualitativas indicaram presença de alguns gêneros patogênicos ${ }^{(11)}$. Os limites foram comparados à RE $n \circ 9$ da ANVISA, que não é específica para o ambiente em questão. $O$ seu limite de exposição é padronizado para locais climatizados de uso público e coletivo e tem valor fixado em $750 \mathrm{ufc} / \mathrm{m}^{3}$.

No ambiente estudado ocorrem poucos procedimentos de necropsia, atividade ligada aos LAP, que é indicada pela literatura como sendo a que envolve maiores riscos de contaminação biológica. Durante a fase de amostragem, não foi possível realizar coleta nesse setor, o que indica a necessidade de novas amostragens no local e em outros LAP, onde a atividade laboral seja mais intensa ${ }^{(11)}$.

A não realização de análise quantitativa de bactérias nos pontos 1, 2, 3 e 4 em comparação aos micro-organismos identificados nos pontos 5 e 6 sugere a necessidade de novas amostragens no local, já que no ponto 5 , representado pelo corredor, foi encontrado o gênero Staphylococcus e no ponto 6 (arquivo morto), os gêneros Staphylococcus e Pseudomonas. Não foi realizada medição de bactérias nos demais pontos selecionados.

O risco biológico nesse ambiente é contínuo, o que torna indispensáveis a correção das irregularidades encontradas e a instalação de equipamentos que garantam a proteção contra contaminação por riscos biológicos.

\section{Conclusão}

Por meio da avaliação ambiental realizada, constatou-se a presença de riscos diversificados no LAP. Com relação aos riscos químicos, o xileno foi encontrado em baixas concentrações. No entanto, dependendo da atividade realizada, há possibilidade de ocorrência de picos de elevada concentração, o que justifica a necessidade de implantação de medidas preventivas.

O formaldeído, porém, foi encontrado em concentrações elevadas, acima do padrão de exposição nacional, considerado permissivo. A concentração de formaldeído medida em duas amostras na sala de clivagem ultrapassou o valor máximo estabelecido pela NR-15, caracterizando risco grave e iminente, o que implica a necessidade de paralisação das atividades até que ocorra a implementação das medidas de controle que possibilitem o restabelecimento de uma situação de normalidade. 
Para reduzir os riscos ambientais foram sugeridas medidas de ordem administrativa, de engenharia e de proteção individual. Como medidas de engenharia, climatização artificial e exaustão mecânica são indicações da RDC no 50/2002, atualizada pela Resolução RDC no 307, de 14 de novembro de 2002, para a atividade de anatomia patológica.

Os LAP devem possuir sistema de ventilação local exaustora eficiente, o que constitui medida de controle na fonte. Sugerem-se como medidas de controle no meio o estudo e a implantação de sistema de ventilação geral diluidora em todas as salas onde há presença de formaldeído, sendo o sistema de exaustão da sala de clivagem separado dos sistemas das demais salas. É indispensável que ocorra manutenção dos sistemas de ar condicionado, que podem ser disseminadores de grande variedade de agentes químicos e biológicos, além de investimentos para adequação das instalações a padrões de qualidade ambiental satisfatórios.
Verificou-se a necessidade de elaboração de normas técnicas que disponham sobre padrões referenciais de qualidade do ar interior e monitoramento dos riscos químicos e biológicos em LAP.

Os dados obtidos com a pesquisa apontaram a necessidade de investigação de outros LAP da rede pública e, em especial, de necropsia, tais como os Institutos Médicos Legais (IMLs), no que tange a qualidade do ar e a contaminação por agentes químicos e biológicos.

É indispensável que sejam realizadas pesquisas comparativas entre diferentes laboratórios e que análises quantitativas e qualitativas mais abrangentes sejam realizadas, a fim de se traçar um perfil das atuais condições dos LAP climatizados públicos e privados.

A obtenção de melhores condições ambientais em LAP dependerá da adoção de medidas preventivas, prevalecendo tais medidas sobre as corretivas.

\section{Referências}

1. ASSOCIAÇÃO BRASILEIRA DE NORMAS TÉCNICAS (ABNT). Norma Regulamentadora NBR 5413, Iluminância de Interiores, Disponível em: http://www. labcon.ufsc.br/anexos/13.pdf. Acesso em 15 jun.2008.

2. Bettini, D. R. Qualidade do ar em laboratório climatizado de anatomia patológica. Avaliação de agentes químicos: Faculdade de Ciências Médicas da UERJ. Rio de Janeiro, 2006. Dissertação (Mestrado) - Programa de Pós-Graduação em Engenharia Ambiental, Faculdade de Engenharia, Universidade do Estado do Rio de Janeiro.

3. BRASIL1 - Ministério da Saúde. Agência Nacional de Vigilância Sanitária (ANVISA). Resolução no 9 de 16 de janeiro de 2003. Dispõe sobre padrões referenciais de qualidade do ar interior em ambientes climatizados artificialmente de uso público e coletivo. Disponível em: http://www.anvisa.gov.br/Legis/index.htm. Acesso em: 10 jan. 2006.

4. BRASIL2 - Ministério do Trabalho e Emprego. Portaria no 3.214 de 8 de junho de 1978 - Aprova as Normas Regulamentadoras relativas à Segurança e Medicina do Trabalho. Disponível em: http://www3.dataprev.gov. br/SISLEX/paginas/63/mte/1978/3214.htm. Acesso em: 10 jan. 2006.

5. BRASIL3 - Ministério do Trabalho e Emprego. Norma Regulamentadora n7 - Programa de Controle Médico de Saúde Ocupacional (PCMSO). Disponível em: http:// www.mte.gov.br/legislacao/normas_regulamentadoras/ nr_07_at.pdf. Acesso em 10 jan. 2006.

6. BRASIL4 - Ministério da Saúde. Agência Nacional de Vigilância Sanitária (ANVISA). Resolução RDC ㄲo 50, de
21 de fevereiro de 2002. "Dispõe sobre o Regulamento Técnico para planejamento, programação, elaboração e avaliação de projetos físicos de estabelecimentos assistenciais de saúde". Disponível em: http://www. anvisa.gov.br/legis. Acesso em: 1 maio 2004.

7. BRASIL5 - Ministério da Saúde. Agência Nacional de Vigilância Sanitária (ANVISA). Resolução RDC no 307, de 14 de novembro de 2002. "Altera a Resolução RDC no 50 de 21 de fevereiro de 2002. "Dispõe sobre o Regulamento Técnico para planejamento, programação, elaboração e avaliação de projetos físicos de estabelecimentos assistenciais de saúde". Disponível em: http://www.anvisa.gov.br/legis. Acesso em: 1 jan. 2004.

8. BRASIL6 - Ministério da Saúde. Agência Nacional de Vigilância Sanitária (ANVISA). Portaria no 3.523/GM, de 28 de agosto de 1998, "Aprova o Regulamento Técnico contendo medidas básicas referentes aos procedimentos de verificação visual do estado de limpeza, remoção de sujidades por métodos físicos e manutenção do estado de integridade e eficiência de todos os componentes dos sistemas de climatização, para garantir a qualidade do ar de interiores e prevenção de riscos à saúde dos ocupantes de ambientes climatizados". Disponível em: http://www.anvisa.gov.br/ legis/portarias/3523_98.htm. Acesso em: 20 jan.2006.

9. BRASIL7 - Ministério do Trabalho e Emprego. Norma Regulamentadora no 15 . Atividades e Operações Insalubres, Disponível em: http://www.mte.gov.br/ legislacao/normas_regulamentadoras/nr_15.pdf. Acesso em 15 fev.2009. 
10. BURTON, J. L. Health and safety at necropsy. Journal of Clinical Pathology, v. 56, n. 4, p. 254-60, 2003. Disponível em: http://www.ncbi.nlm.nih.gov/pubmed/. Acesso em: 18 mar. 2008.

11. Franklin, S. L. A. qualidade do ar em laboratórios de anatomia patológica climatizados - avaliação dos agentes biológicos. Rio de Janeiro, 2006. Dissertação (Mestrado) - Programa de Pós-Graduação em Engenharia Ambiental, Faculdade de Engenharia, Universidade do Estado do Rio de Janeiro.

12. HÖKERBERG, Y. A. M. et al. O processo de construção de mapas de risco em um hospital público. Ciência e Saúde Coletiva, v. 11, n. 2, p. 1-21, 2006.

13. INTERNATIONAL AGENCY FOR RESEARCH ON CANCER (IARC). IARC monographs on the evaluation of carcinogenic risks to humans. Disponível em: http:// monographs.iarc.fr/ENG/Preamble/index.php. Acesso em: 06 mar. 2006.

14. INTERNATIONAL AGENCY FOR RESEARCH ON CANCER (IARC). IARC monographs on the evaluation of carcinogenic risks to humans - formaldehyde, 2-butoxyethanol and 1-tert-butoxypropan-2-ol., v. 88,
2006. Disponível em:http://monographs.iarc.fr/ENG/ Monographs/vol88/.

15. MATTOS, U. O.; QUEIROZ, A. R. Mapa de risco, In: Biossegurança: uma abordagem multidisciplinar. Rio de Janeiro: FioCruz, 1996.

16. MONTENEGRO, M. R.; FRANCO, M. Patologia: processos gerais, 3. ed. Rio de Janeiro: Livraria Atheneu, 1992.

17. MORAWSKA, L. et al. Particulate matter in the hospital environment, Indoor Air, vol. 8, p. 285-94, 1998.

18. NATIONAL INSTITUTE FOR OCCUPATIONAL SAFETY AND HEALTH (NIOSH). Pocket Guide to Chemical Hazards. USA: 2001. Disponível em: http://www.cdc. gov/niosh/npg/. Acesso em: 10 fev. 2006.

19. OCCUPATIONAL SAFETY \& HEALTH ADMINISTRATION (OSHA) - Chemical sampling information. Disponível em: http://www.osha.gov/dts/chemicalsampling/data/ CH_249110.html. Acesso em: 3 jul. 2004.

20. SAAD, I. F. S. D. et al. Contaminantes de origem biológica veiculados pelo ar. In: Limites de exposição (TLVS) para substâncias químicas e agentes físicos e índices biológicos de exposição (BE/s). São Paulo: Associação Brasileira de Higienistas Ocupacionais (ABHO), 2003. 